\title{
On a New Basic Concept and Topological Invariant
}

\author{
Franck XIA \\ Department of Information Systems \\ University of Macao, P.O. Box 3001 Macao \\ E-mail: fbafx@uealab.umac.mo
}

\begin{abstract}
Component is one of the most basic topological concepts for digital images. However, there is still few simple method, to date, permitting to distinguish holes from external borders. Such theoretic difficulty on components leads to quite complex and unnatural algorithms on component analysis. In this paper, the author proves that there exists, in 2D images, a basic topological invariant called total curvature. The total curvature on a border is the sum of local curvature representing the external angle of polygon vertices. We have discovered an important binary feature of this invariant: it equals to $2 \pi$ for external contours and $-2 \pi$ for hole contours, independent of the orientation of contour tracing. The binary characteristics of total curvature reveals that external contours are global convex despite of local concavities and hole contours are global concave despite of local convexities. Therefore hole and external contours can be unified as total convex and total concave borders. Classifying all the borders of images by total curvature provides directly the number of both components and holes and it can be calculated easily in digital images.
\end{abstract}

\section{Introduction}

Digital topology has been extensively explored in the past [1]. The effort was concentrated on a graph-theory approach which is widely accepted in computer science community. Digital topology provides a theoretic basis for various elementary operations in image processing such as thinning, border following, contour filling, object counting and extraction and the like.

Two concepts are fundamental in digital topology: connectedness and component. The problem of connectedness has been extensively studied in the past through skeletonization. Our interest in this paper is focused on the properties of two dimensional connected components, which is not well explored, according to the author, comparing to the abundant literature on connectedness. Undoubtedly, component analysis is an important issue in image processing, since components are basic elements of images and they represent generally objects or parts of object. The research on this issue, basically on parallel component labelling, is quite active [2].

Regions or connected components are entirely enclosed by their external contour in single connection case or with included holes in case of multiply connection. Intuitively, if we can detect all the borders and distinguish outer contours from hole contours in images, then the description of components is straightforward. In digital topology, people generally use Euler characteristics to describe component since Euler number equals to the number of components minus the number of holes.

The problem is that from computational viewpoint, Euler number is not really helpful for component analysis. This is because even to determine the number of components in images, we must determine first the number of holes and then deduce 
the number of components from Euler number. But detecting holes in an images is an unsolved problem, in practice, although the concept of hole is intuitive $Z^{2}$.

Until very recently, there was no satisfactory method presented in literature detecting directly inner contours. Suzuki and Abe proposed an algorithm being able to describe the nested structure of borders and hence differentiate outer contour from holes indirectly [3]. The differentiation of components from holes is not based on border's characteristics but on the nested structure of whole image: background surrounds components, components may include holes and holes may, in turn, surround objects, etc. [4]. Even to extract a small component, the whole image must be analysed first. Another tentative was based on mathematical morphology which has a serious drawback to change the topological features of images [5].

The only theoretical work on holes and their detection was carried out by Lee, Poston and Rosenfeld in 1991 [6]. The idea consists of counting the transition number of normal vector on contours in a given direction to distinguish holes from components. Their work provides the first theoretic method for component analysis. The problem revealed is that the normal vector of boundary is intrinsically discontinuous. Therefore they suggested to use a continuous smoothing function replacing the digital lattice and normal vectors are calculated with the smoothing function. Counting the winding number cannot be realized directly with digital images, unfortunately.

In general, due to the barrier to distinguish external borders from hole borders, component analysis algorithms remain generally unnatural and complex. A typical example is sequential component labelling through raster scanning, which requires either time consuming iterative label propagation or using complex and memory consuming equivalence relationship [7].

\section{Proposition}

In this communication, we prove that total curvature - the sum of local curvature on borders representing the complement of internal angles at border vertices - is a very attractive and basic topological invariant. The real important discovery is that, in fact, the total curvature of an arbitrary borders has only two constant values $2 \pi$ or $-2 \pi$, relying on whether the border is external or internal.

The binary characteristics of total curvature reveals the intrinsic property of contours: external contours are global convex despite of local concavity and hole contours are global concave despite of local convexity. Therefore external contours and hole contours can be unified as total convex and total concave borders based on the concept of total curvature. Classifying all the borders of images by total curvature provides directly the number of both components and holes. The computation of total curvature allows a very simple separation of outer contours from hole contours. Applying this method to digital images is extremely simple.

We start to examine total curvature of polygons and then apply the invariant to digital images. Computation results are given to illustrate the simplicity and usefulness of the invariant.

\section{Topological Invariant of Polygon}

We will describe in this section the invariant property of single closed polygons, since all digital images can be described by single closed polygon (we denote simply 
by polygon in this paper). Discussing the invariant of polygons provides an universal theory for 2D digital images which is completely independent of neighborhood used. This is a major advantage. The proof of the following results can be found in [8]. Definition 3.1: Local curvature $\Delta \alpha_{\mathrm{i}}$ at vertex $\mathrm{A}_{\mathrm{i}}$ of a polygon equals to $\pi-\beta_{i}$, with $\beta_{i}$ the interior angle of the polygon at $A_{i}$ (Fig. 1).

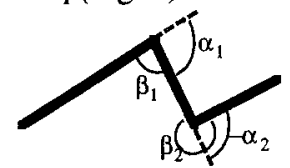

Figure 1: Local Curvature in $\mathbf{R}^{2}$

Definition 3.2: For any polygon, the total curvature is the sum of local curvatures on all the vertices $\sum_{i} \Delta \alpha_{i}$.

Remark: the local curvature is independent of the direction in which all the vertices of the polygon are traversed. In fact the local curvature at $A_{i}$ is positive if the internal angle $A_{i}$ is convex and negative if concave.

Proposition 3.3: For any convex polygon of $M$ edges, the sum of interior angles $\sum_{i} \beta_{i}(P)=(\mathrm{M}-2) \pi$.

Denote $\beta_{i}(P)$ the $\mathrm{i}^{\text {th }}$ interior angle of $\mathbf{P}$ and $\beta_{j}^{c}(P)$ the $\mathrm{j}^{\text {th }}$ concave interior angle of $\mathrm{P}$, with $\beta_{j}^{c}(P)>\pi$.

Lemma 3.4: Any polygon $\mathrm{P}$ of $\mathrm{N}$ concave interior angle $\left(\sum_{i} \beta_{i}^{c}\left(P_{1}\right)=\mathrm{N} \geq 1\right)$ can be divided into two parts $P_{1} \& P_{2}$, by a segment starting from a vertex of $P$, such that

1) the number of concave interior angles in each polygon reduced at least 1 :

$\sum_{i} \beta_{i}^{c}\left(P_{1}\right) \leq \mathrm{N}-1$ and $\sum_{i} \beta_{i}^{c}\left(P_{2}\right) \leq \mathrm{N}-1$;

2) the sum of interior angle of $P_{1}, P_{2}$ and $P$ have the following relation:

$\sum_{i} \beta_{i}\left(P_{1}\right)+\sum_{i} \beta_{i}\left(P_{2}\right)-\gamma \pi=\sum_{i} \beta_{i}(P)$ where

$\gamma=0$ if the segment does not cut any edge of $P$ and no new vertex is generated and

$\gamma=1$ if the segment cuts an edge of $P$ and a new vertex is generated.

Lemma 3.4 is essential for the proof of theorem 3.5.

Theorem 3.5: For any polygon of $M$ edges, the sum of its interior angles is (M-2) $\pi$.

Theorem 3.6: For any polygon $P$, its total curvature is $2 \pi$.

This theorem is fundamental for our method and evident by theorem 3.5 .

Corollary 3.7: For a special sort of polygon in which two different polygon paths coincide, its total curvature is $2 \pi$.

This theorem is very useful for discussing holes. It allows us to cut a region with a open polygon inside the region and discuss the two sides of the same open polygon.

Proposition 3.8: For any open polygon $\mathrm{p}_{0} \mathrm{p}_{1} \mathrm{p}_{2} \ldots \mathrm{p}_{\mathrm{n}-1} \mathrm{p}_{\mathrm{n}}$, the sum of vertex curvatures calculated from both sides of the open polygon excluding the two extremities is zero.

Corollary 3.7 and proposition 3.8 are useful for theorem 3.9 .

Theorem 3.9: For any multiply connected region bounded by two polygons, the total curvature of its hole polygon equals to $-2 \pi$.

In topology, vertices, sides and surfaces are major concerns as they have practical meaning in many application fields. In contrary, the property of boundary has not attracted the interest of topologists. Here we provide, by total curvature, a simple way to distinguish external borders from holes of polygon. Note that the distinction of hole 
borders from external borders is not at all related to the direction of border tracing. It is based on the intrinsic global convexity and global concavity of borders.

\section{Contour Invariant in Digital Images}

When applying the new property of polygon invariànce to digital images, we follow basic definitions on discrete geometry described in [1].

Definition 4.1: Let $\mathrm{P}_{\mathrm{i}-1}, \mathrm{P}_{\mathrm{i}}$ and $\mathrm{P}_{\mathrm{i}+1}$ be three consecutive contour pixels and separate its $\mathrm{j}$-neighbors into object side and background side. Let $\angle \mathrm{P}_{\mathrm{i}-1} \mathrm{P}_{\mathrm{i}} \mathrm{P}_{\mathrm{i}+1}$ be the interior angle (in object side) at $P_{i}$. Local curvature at $P_{i} \mathbf{L C}\left(P_{i}\right)$ represents the complementary angle of $\angle \mathrm{P}_{\mathrm{i}-1} \mathrm{P}_{\mathrm{i}} \mathrm{P}_{\mathrm{i}+1}: \pi-\angle \mathrm{P}_{\mathrm{i}-1} \mathrm{P}_{\mathrm{i}} \mathrm{P}_{\mathrm{i}+1}$ (Fig. 2). Note shaded pixels are interior points.

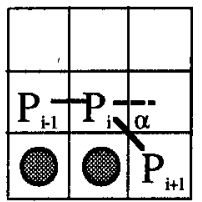

(a)

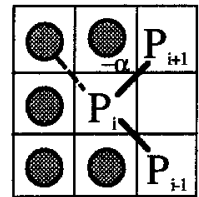

(b)

Figure 2: Local curvature in 8-neighbors.

The value of local curvature $\operatorname{LC}\left(\mathrm{P}_{\mathrm{i}}\right) \geq 0$ if $\angle \mathrm{P}_{\mathrm{i}-1} \mathrm{P}_{\mathrm{i}} \mathrm{P}_{\mathrm{i}+1}$ is convex (Fig.2.a) or $\operatorname{LC}\left(\mathrm{P}_{\mathrm{i}}\right.$ ) $<0$ when $\angle \mathrm{P}_{\mathrm{i}-1} \mathrm{P}_{\mathrm{i}} \mathrm{P}_{\mathrm{i}+1}$ is concave. (Fig. 2.b) As local curvature is a new concept in digital images, we illustrate in Figure 3 examples of local curvature in 8-neighbors. By the definition of contour, $\mathbf{L C}$ may have discrete values $\{-2,-1,0,1,2,3,4\}$. In the figure, dark shaded circles designate object/contour pixels, clear shaded circles stand for the current edge points, and the numeric in center represents $\mathbf{L C}$ at these points. For simplicity, we omit those that can be obtained by rotation of multiples of $90^{\circ}$.
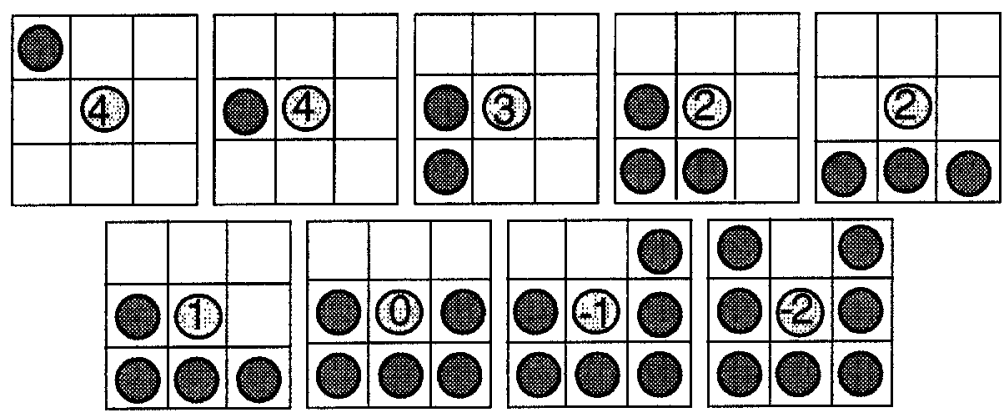

Figure 3: configurations of different local curvatures.

Definition 4.2: Total curvature $\mathbf{T C}(\partial \mathbf{C})$ is the sum of local curvature $\mathbf{L C}$ on a contour $\partial \mathbf{C}$ and denoted by $\sum_{i} L C\left(P_{i} \mid P_{i} \in \partial \mathbf{C}\right)$.

A special case is an isolated object point, since the definition of local curvature requires at least two connected points. We define the total curvature of an isolated point is $2 \pi$. The problem does not exist for hole contours since the minimum perimeter of hole contours is 4 corresponding to a hole of isolated pixel.

Corollary 4.3: In digital images, $\operatorname{TC}\left(\partial \mathrm{C}^{+}\right)=-\mathrm{TC}\left(\partial \mathrm{C}^{-}\right)=\mathrm{N}$, where $\mathrm{N}$ equals 8 for rectangular tessellation and 6 for hexagonal tessellation . 


\section{Result}

Without loss of generality for sequential processing, we adopt the left-hand convention to trace all the borders of multiple connected regions. But in fact, the definitions of local curvature and total curvature are independent of contour tracing orientation.

As computational illustration, we present results of external and hole contour distinction through a very simple algorithm. The algorithm scans once the whole image to find out all the contours - both outer contours and hole contours, by a classical contour following procedure. Contours are encoded with Freeman direction codes and the difference of two consecutive codes is calculated during contour tracing which provides directly the local curvature of contour pixels. The total curvature is obtained simply by summing local curvatures. It is then used to classify outer contours and hole contours. The algorithm is extremely simple.

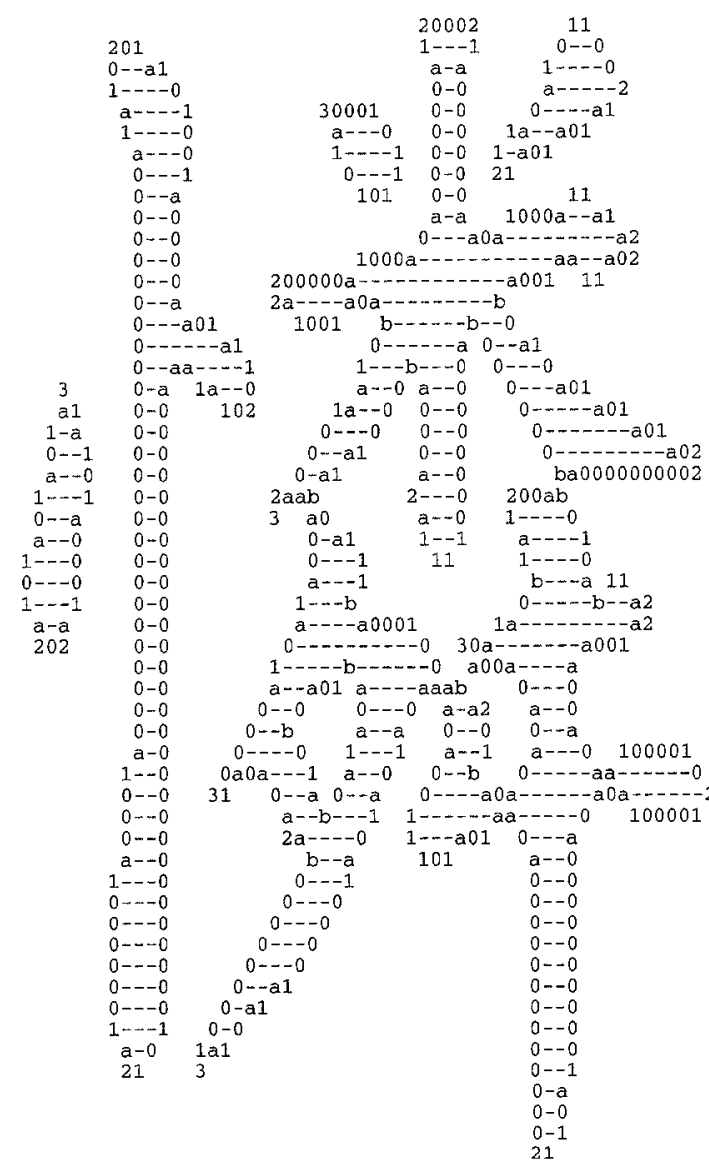

Contour No.1: Perimeter 255, Total Curvature $=8=>$ outer contour
Contour No.2: Perimeter 22, Total Curvature $=8 \Rightarrow$ outer contour
Contour No.3: Perimeter 111, Total Curvature $=8 \Rightarrow$ outer contour
Contour No.4: Perimeter 14, Total Curvature $=8 \Rightarrow$ outer contour
Contour No.5: Perimeter 67, Total Curvature $=-8=>$ hole contour
Contour No.6: Perimeter 26, Total Curvature $=8 \Rightarrow$ outer contour
Contour No.7: Perimeter 16, Total Curvature $=-8 \Rightarrow>$ hole contour
Contour No.8: Perimeter 18, Total Curvature $=-8=>$ hole contour

Figure 4: Components with distinguished outer and hole contours 
Figure 4 illustrates the resulting images in which $a$ and $b$ represent local curvature value -1 and -2 on contour pixels.

A practical advantage of the proposed method to evaluate topological property is that it may insensitive to noise. This is because the perimeter of contours provides the information on the size of components or holes. Depending on applications, small components and holes can be ignored during processing to provide robust topological property.

\section{Conclusion}

In digital space, there is still few simple method, to date, allowing to distinguish holes from other contours. Consequently, the difficulty leads to quite complex and unnatural algorithms in image analysis. In this paper, a novel invariant called total curvature has been presented. The total curvature of borders has an important binary characteristics : It equals to $2 \pi$ for external contours and $-2 \pi$ for hole contours. This means that borders of components are global convex and borders surrounding holes are global concave. The interesting feature can be used to distinguish external contours from hole contours. The application of total curvature is straightforward in digital space. The distinction of holes from external contours is of a great importance, since regions or connected components can be entirely determined by external contours and holes. Thanks to the proposed invariant, a new approach of image analysis is available and methodology of image analysis can be greatly simplified in terms of computational and algorithmic complexity. A very simple algorithm can be derived using the invariant property of border - total curvature to detect, extract and describe connected components without labelling.

\section{Reference}

1. Kong T.Y. and Rosenfeld A., Digital topology: Introduction and survey, CVGIP, vol. 48, 1989, 357-393

2. Alnuweiri H.M. and Prasanna V.K., Parallel architectures and algorithms for image component labeling, IEEE Trans. PAMI, Vol. 14, No. 10, 1992, 1014-1034

3. Suzuki S. and Abe K., Topological structural analysis of digital binary images by border following, CVGIP, vol. 30, 1985, 32-46

4. Rosenfeld A., Picture Languages, Academic Press, New York, 1979

5. Qian K. and Bhattacharya P., Determining holes and connectivity in binay images, Comput. \& Graphics, vol. 16, no. 3, 1992, 283-288

6. Lee C.N., Poston T. and Rosenfeld A., Winding and Euler numbers for 2D and 3D digital images, CVGIP: Graphical Model and Image Processing, vol. 55, no. 1, 1993, 20-47

7. Haralick R., Some neighborhood operators, Real-Time Parallel Computing Image Analysis, Onoe M., Preston K. Jr. \& Rosenfeld A. (eds.), Plenum, New York, 1981

8. Xia F., Total Curvature - a New and Fundamental Concept and Topological Invariant for Digital Images, to appear in IEEE Trans. PAMI 